\title{
Differential effects of natural Curcumin and chemically modified curcumin on inflammation and bone resorption in model of experimental periodontitis
}

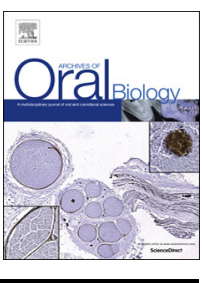

\author{
Fabiana Almeida Curylofo-Zotti ${ }^{\mathrm{a}, 1}$, Muna S. Elburki ${ }^{\mathrm{b}}$, Priscilla Aparecida Oliveira ${ }^{\mathrm{c}}$, \\ Carlos Rossa Junior ${ }^{\mathrm{a}}$, Morgana Rodrigues Guimarães-Stabili ${ }^{\mathrm{a}, *}$ \\ ${ }^{a}$ Department of Restorative Dentistry School of Dentistry of Riberão Preto University of São Paulo Riberão Preto SP Brazil \\ ${ }^{b}$ Department of Oral Biology and Pathology, School of Dental Medicine, SUNY at Stony Brook, Stony Brook, USA \\ ${ }^{\mathrm{c}}$ Laboratory of Histology and Embryology, School of Dentistry at Araraquara, UNESP, Araraquara, SP, Brazil \\ ${ }^{\mathrm{d}}$ Department of Chemistry and Pharmacological Sciences, SUNY at Stony Brook, Stony Brook, USA
} Paulo Sérgio Cerri ${ }^{\mathrm{c}}$, Leandro Alves Santos ${ }^{\mathrm{a}}$, Hsi-Ming Lee ${ }^{\mathrm{b}}$, Francis Johnson ${ }^{\mathrm{d}}$, Lorne M. Golub ${ }^{\mathrm{b}}$,

\section{A R T I C L E I N F O}

\section{Keywords:}

Bone resorption

Chemically modified curcumin (CMC2.24)

Periodontal disease

Lipopolysaccharide

Inflammation

\begin{abstract}
A B S T R A C T
Objective: The purpose of this study was to compare the effects of the oral administration of natural curcumin and a chemically modified curcumin (CMC2.24) on osteoclast-mediated bone resorption, apoptosis, and inflammation in a murine model of experimental periodontal disease.

Design: Fifty male rats were distributed among the following treatment groups: (i) $2 \%$ carboxymethylcellulose, (ii) CMC2.24 $30 \mathrm{mg} / \mathrm{kg}$ body weight, (iii) Curcumin $100 \mathrm{mg} / \mathrm{kg}$ body weight and (iv) no treatment. Compounds were administered daily by oral intubation over a 15-day period of time. Periodontal disease was induced by injections of LPS (lipopolysaccharide) into the gingival tissues three times per week. Contralateral sides were injected with the same volume of PBS (phosphate buffered saline) vehicle. After 15 days, hemimaxillae and gingival tissues were harvested. Bone resorption was assessed by $\mu \mathrm{CT}$ (microcomputer tomography). Formalinfixed, paraffin embedded histological sections were stained with haematoxylin/eosin $(\mathrm{H} / \mathrm{E})$ for the assessment of cellular infiltrate or subjected to immunohistochemistry for detecting TRAP (tartrate-resistant acid phosphatase)-positive cells and caspase-3. Apoptosis was assessed in the gingival tissues by DNA fragmentation.

Results: CMC2.24 and curcumin caused a significant reduction of the inflammatory cell infiltrate, however $\mu$ CT analysis showed that only CMC2.24 reduced bone resorption and the number of TRAP-positive multinucleated cells (osteoclasts). Curcumin, but not CMC2.24, significantly reduced the number of apoptotic cells in the gingival tissues and of osteocytes in the alveolar bone crest.

Conclusions: The results suggest that CMC2.24 and curcumin inhibit inflammation by different mechanisms, but only CMC2.24 was capable of reducing alveolar bone resorption in the LPS-induced model of periodontitis.
\end{abstract}

\section{Introduction}

Natural curcumin (diferuloylmethane) is a hydrophobic polyphenol composed of a mixture of three curcuminoids: curcumin, demethoxycurcumin and bisdemethoxycurcumin with various biological activities reported (Shehzad, Park, Lee, \& Lee, 2013). Diverse studies report on anti-inflammatory, anti-microbial and anti-neoplastic properties of curcumin in diverse conditions such as diabetes, cancer, auto-immune conditions and chronic inflammatory conditions including Crohn's disease and rheumatoid arthritis. Despite the promising perspectives (Di Martino et al., 2017; Kumar, Ahuja, Ali, \& Baboota, 2010), clinical use of curcumin is limited because of its poor absorption in the gastrointestinal tract, short plasma half-life and low bioavailability after oral administration (Anand, Kunnumakkara, Newman, \& Aggarwal, 2007; Shoba et al., 1998).

Based on the reports of potent biological activities and on studies indicating its safety and virtual absence of unwanted side effects (Lao et al., 2006; Vernillo, Ramamurthy, Golub, \& Rifkin, 1994), there is great interest in developing synthetic analogues, with a defined and consistent chemical composition and improved pharmacological properties. The so-called chemically modified curcumins (CMC) combine low toxicity and potent inhibitory activity of matrix metalloproteinases (MMPs) (Zhang, Golub, Johnson, \& Wishnia, 2012), properties that justify an assessment in vivo models. CMCs are part of a class of

\footnotetext{
* Corresponding author.

E-mail address: morganaguimaraes@foar.unesp.br (M.R. Guimarães-Stabili).

${ }^{1}$ Current address: Department of Restorative Dentistry, School of Dentistry of Ribeirão Preto, University of São Paulo, Ribeirão Preto, SP, Brazil.
} 
polyenolic Zinc-binding inhibitors able to inhibit both MMPs and cytokines. These compounds were originally developed as 1,3-bis ketone synthetic analogues, based on the fundamental role of $\beta$-ketone as a zinc-binding region (Golub, Suomalainen, \& Sorsa, 1992). The natural and some early chemically-modified curcumins are di-ketonic; however the compound tested in this and our more recent studies (Elburki, Rossa et al., 2017; Elburki, Moore et al., 2017; Elburki et al., 2014; Zhang et al., 2012) are tri-ketonic with stronger $\mathrm{Zn}^{++}$binding and MMP-inhibitory properties. This tri-ketonic CMC has a phenyl-aminocarbonyl group at carbon 4 and demonstrates higher water solubility than the natural compound (unmodified natural curcumin). The potency of different formulations of newer CMCs as inhibitors of MMPs was evaluated in vitro and CMC2.24, in particular, had the lowest IC50 values (Zhang et al., 2012). In an in vitro model of inflammationmediated cartilage destruction, CMC2.24 inhibited cartilage degradation by $96 \%$ in comparison with the vehicle control, whereas natural (unmodified) curcumin had no effect (Elburki, Rossa et al., 2017).

Evidence reported in the literature and studies by our research group using natural curcumin and CMC2.24 demonstrate a significant effect in the inhibition of inflammation and bone resorption (Correa et al., 2017; Elburki, Rossa et al., 2017; Elburki, Moore et al., 2017; Elburki et al., 2014; Guimaraes et al., 2011; Guimaraes et al., 2012; Zhou et al., 2013), which are hallmark characteristics of experimental models of periodontal disease and also of the clinical condition in humans (Dentino, Lee, Mailhot, \& Hefti, 2013; Graves, Kang, Andriankaja, Wada, \& Rossa, 2012; Hajishengallis, Lamont, \& Graves, 2015). In spite of a significant inhibition of the inflammatory infiltrate and potent reduction of various inflammatory mediators, oral administration of curcumin had no effect on inflammatory bone resorption (Guimaraes et al., 2012), a critical and usually irreversible feature of destructive periodontal disease. Even studies that report significant curcumin-induced inhibition of bone resorption in periodontal disease models, the decrease in bone resorption severity was less than $10 \%$ in comparison with the vehicle control group (Correa et al., 2017; Zhou et al., 2013). On the other hand, an in vivo study showed that systemic administration of CMC2.24 reduced LPS-induced bone resorption in rats by more than 20\% (Elburki, Moore et al., 2017). However, these studies were performed independently and there is no direct comparison between natural curcumin and CMC2.24 in a simultaneously executed experiment.

Based on data suggesting increased biological potency and superior pharmacological properties of CMC2.24 in comparison with natural curcumin, this study now describes and compares, for the first time in parallel experiments carried out simultaneously, the effects of the oral administration of natural curcumin and CMC2.24 in a murine model of LPS-induced periodontal disease. The outcomes of interest were: bone resorption, inflammation, osteoclastogenesis and apoptosis.

\section{Materials and methods}

\subsection{Experimental design}

The experimental protocol was approved by the Ethical Committee for Animal Use (CEUA) of the School of Dentistry at Araraquara UNESP (license number 12/2011) and performed in accordance with the guidelines from the Brazilian College for Animal Experimentation (COBEA). Fifty male Holtzman rats (Rattus norvegicus albinus, Holtzman) of 10 to 14 weeks of age and weighing between 150 and $200 \mathrm{~g}$ were used in this study. Animals were kept in polypropylene cages in a room with controlled temperature $(21 \pm 1 \mathrm{C})$ and humidity $(65-70 \%)$ and a $12 \mathrm{~h}$ light-dark cycle. The rats were fed standard rat chow (Labina/ Purina) and water ad libitum. All animals were submitted to injections on the palatal aspect of the first molars bilaterally: LPS was injected on left side and PBS on the right side (Elburki, Rossa et al., 2017; Elburki, Moore et al., 2017; Elburki et al., 2014; Guimaraes et al., 2012). After inhalation anaesthesia, $30 \mu \mathrm{g}$ of lipopolysaccharide from Escherichia coli (strain O55:B5; Sigma Chemical Co., St Louis, MO, USA) diluted in PBS were injected into the palatal gingiva ( $3 \mathrm{uL}$ volume per injection) using a $10 \mu \mathrm{L}$ Hamilton-type microsyringe (Agilent). Control sides were injected with the same volume of PBS vehicle. Injections were performed three times per week for 15 days between the upper first and second molars using syringes and needles dedicated for either LPS or PBS. The animals were randomly assigned to the following four experimental groups ( $\mathrm{n}=10$ animals/group) according to the compound administered systemically: (i) 2\% carboxymethylcellulose (CMC2.24 vehicle control), (ii) CMC2.24 $30 \mathrm{mg} / \mathrm{kg}$ body weight, (iii) Curcumin $100 \mathrm{mg}$ / $\mathrm{kg}$ body weight and (iv) no treatment. CMC2.24 was synthesized at the laboratories of the Chemistry Department, State University of New York (SUNY) at Stony Brook (Stony Brook, NY, USA) and natural curcumin was obtained commercially (Sigma-Aldrich Co. cat\# C1386, Lot\# $081 \mathrm{M} 1611 \mathrm{~V}$ ). The doses of $30 \mathrm{mg} / \mathrm{kg}$ CMC2.24 and $100 \mathrm{mg} / \mathrm{kg}$ curcumin were based in our previous studies showing that both doses are effective in reducing inflammatory mediators production and inflammatory infiltrate in gingival tissue of rats with experimentally-induced periodontitis (Elburki, Rossa et al., 2017; Elburki, Moore et al., 2017; Elburki et al., 2014; Guimaraes et al., 2012; Guimaraes et al., 2011). A group treated only with corn oil (curcumin vehicle control) was not included in this study because data from our previous studies showed no effect of lipid vehicle on bone loss and inflammation (Guimaraes et al., 2011; Correa et al., 2017). Compounds were administered daily during 15 days by oral intubation beginning $24 \mathrm{~h}$ after the start of local PBS and LPS injections in the protocol for induction of experimental periodontal disease. Animals were euthanized by cervical dislocation under inhalation anaesthesia and the hemi-maxillae were carefully dissected. Gingival soft tissue (approximately $1.5 \mathrm{~mm}$ in the frontal plane $\times 3 \mathrm{~mm}$ in the sagittal plane) adjacent to the upper first molars were carefully dissected from 5 samples from each experimental condition, immediately flash-frozen in liquid nitrogen and subsequently stored at $-80 \mathrm{C}$ until the moment of total protein extraction. The other 5 samples from each experimental condition were subjected to $10 \%$ buffered formalin fixation and subsequent histological processing for paraffin embedding (as described below) and used in histological and immunohistochemical analysis.

\subsection{Microcomputer tomography (uCT)}

All hemi-maxillae (with and without soft tissues) were scanned on a microcomputer tomograph (Skyscan 1176, SkyScan Aartselaar, Belgium) using $18 \mu \mathrm{m}$ slices. Digital radiographic images of each sample were reconstructed into a three dimensional model and a standardized gray scale value was set to distinguish mineralized from non-mineralized tissues. These three dimensional images were re-oriented on the sagittal, coronal and transversal planes in a standardized manner using anatomical landmarks on molar teeth. A standardized region of interest of $9.72 \mathrm{~mm}^{3}$ was defined, including the first molar, the anterior half of the second molar and extending medially (towards the center of the palate) approximately $1 \mathrm{~mm}$ from the most palatal aspect of the crown of the first molar. All image reconstruction, reorientation and analysis of bone volume/total volume (BV/TV fraction) in the region of interest was performed using the software package of the scanning equipment (NRecon/Dataviewer/CTan/CTvo, Skyscan, Aartselaar, Belgium) by a trained examiner who was not aware of the experimental conditions of each sample.

\subsection{Stereometric and morphometric analysis}

The hemi-maxillae with preserved soft tissues were immersed in $10 \%$ buffered formalin fixative solution for $24 \mathrm{~h}$, washed in running water decalcified in tetrasodium-EDTA aqueous solution $(0.5 \mathrm{M}, \mathrm{pH}$ 8.0) for 2-3 months, under agitation at room temperature. Each specimen consisted of a section containing the three upper molars and the surrounding alveolar process and soft tissues. After inclusion in 
paraffin, $5 \mu \mathrm{m}$ thick semi-serial sections (spacing of $100 \mu \mathrm{m}$ between sections) were obtained in the bucco-lingual (frontal plane) direction and stained with $(\mathrm{H} / \mathrm{E})$ or kept unstained for immunohistochemical analysis. The percentage of collagen fibers and the number of inflammatory cells (neutrophils and mononuclear cells) present in gingival tissue was conducted by a single examiner who was blind to the experimental groups and previously trained. The area of interest was standardized to obtain digital images used in the analysis. Initially, images at $200 \times$ magnification were obtained from the gingival connective tissue located $400 \mu \mathrm{m}$ towards the medial (i.e., center of palate) from the most apical portion of the junctional epithelium of the upper first molar. These images were obtained in a conventional optical microscope using standardized settings for image acquisition (Leica Application Suite 3.8, Wetzlar, Germany). To quantify the collagen fibers on gingival tissue, a grid composed of 10 squares with $10 \mu \mathrm{m}$ sides (area of $100 \mathrm{~m}^{2}$ ) was positioned onto the digital images obtained at $200 \times$. A total of 108 points (intersections) were counted and the percentage of collagen fibers in relation to the total number of points was calculated. To analyze the cellular infiltrate on the gingival tissues, $600 \times$ images of 4 random fields in the $200 \times$ magnification images were evaluated. Cells were distinguished as neutrophils or mononuclear cells by the nuclear morphology. In these analyses, a total of 4 sections per specimen (20 sections for each experimental condition, from 5 different animals) spaced $100 \mu \mathrm{m}$ from each other (antero-posterior plane) were evaluated.

\subsection{Osteoclast count}

Osteoclasts were identified by their morphology, location and positivity for the expression of tartrate-resistant acid phosphatase (TRAP), assessed by immunohistochemistry. Sections mounted on silanized slides were deparaffinized and rehydrated in a decreasing concentrations of ethanol. Endogenous peroxidase was blocked with 3\% hydrogen peroxide for $30 \mathrm{~min}$. To inhibit non-specific interactions of the primary antibody, the sections were incubated with a commercial blocking solution (X0909, Dako - Agilent Technologies) according to the manufacturer's instructions. Slides were washed in distilled water and incubated overnight at 4C with goat polyclonal antibody for TRAP (cat\# sc-30833, Santa Cruz Biotechnology) diluted 1:100 in PBS containing $2 \%$ BSA. Controls included omission of the primary antibody and also incubation with the same concentration of irrelevant IgG raised in goat. After washings in distilled water, the sections were incubated with biotinylated anti-goat antibodies for $30 \mathrm{~min}$, washed, incubated with streptavidin conjugated to HRP and finally with the DAB substrate, according to the protocol of the supplier of the visualization system (LSAB2, Dako - Agilent Technologies). Subsequently, the sections were counterstained with Mayeŕs Hematoxylin and coverslips mounted. Osteoclasts were identified as large, multinucleated ( 3 or more visible nuclei) and TRAP-positive cells, located in the vicinity of the alveolar bone, from the apical region of the palatal root of the first molar to a $400 \mu \mathrm{m}$ distance from the palatal alveolar bone crest towards the midline (center of the palate). This analysis was performed in 4 semi-serial sections (spaced $100 \mu \mathrm{m}$ in the antero-posterior plane) from each specimen (20 sections for each experimental condition) by a trained examiner who was unaware of the experimental condition of the sections.

\subsection{Apoptosis}

Detection of active (cleaved) caspase- 3 in the cells of the gingival tissues was performed by immunohistochemistry. The same general protocol described for detection of TRAP was used, with the inclusion of an antigen retrieval step, performed in citrate buffer $\left(\mathrm{pH} \mathrm{6.0)}\right.$ ) at $95^{\circ} \mathrm{C}$ for $20 \mathrm{~min}$. After blocking of endogenous peroxidase and non-specific interactions of the primary antibody, the sections were washed in distilled water and incubated overnight at 4C with 1:50 dilution (in PBS containing $2 \%$ BSA) of rabbit polyclonal antibodies against rat active caspase-3 (cat\# ab2302, Abcam). After washings in distilled water, the sections were incubated with biotinylated anti-rabbit secondary antibodies for $20 \mathrm{~min}$ and the target protein detection was performed using the streptavidin-DAB system, following the instructions of the supplier of the reagents (LSAB2, Dako - Agilent Technologies). Sections were counterstained with Mayeŕs Hematoxylin and coverslips mounted. Negative controls were sections in which primary antibodies were omitted and sections incubated with serum from normal (non-immunized) rabbits. A total of 15 semi-serial sections (3 from each specimen from 5 different animals in each experimental condition) were assessed and the number of positively-stained cells counted in the area of interest (described in stereometric analysis) by a trained examiner who was unaware of the experimental condition of the sections.

We also assessed cell death in the gingival tissues by DNA fragmentation, a hallmark of end-stage apoptosis, using the terminal deoxynucleotidyl transferase dUTP nick end labeling (TUNEL) method. We used a commercial kit, strictly according to the manufacturer's instructions (Apop-Tag Plus kit, Oncor, Gaithersburg, MD). For identification of cell nuclei, coverslips were mounted on the sections using an acqueous mounting medium containing 2-(4-Amidinophenyl)-6-indolecarbamidine dihydrochloride, 4',6-Diamidino-2-phenylindole dihydrochloride (cat\# F6057, Fluoroshield with DAPI, Sigma-Aldrich). Visualization and counting of stained (TUNEL-positive) cells was performed in 20 semi-serial sections (spaced $100 \mu \mathrm{m}$ in the antero-posterior plane) for each experimental condition (4 sections/animal) on an inverted fluorescence microscope (EVOS fl, AMG Micro Group Inc.) by a trained examiner unaware of the experimental condition of the sections.

\subsection{Statistical analysis}

Data obtained from each experiment were analyzed using GraphPad Prism 5.0 (GraphPad Software Inc., San Diego, CA, USA). The objective of the analysis was to compare the results of each outcome according to the different tested compounds. The Shapiro-Wilk test was used to verify that the data had a normal distribution. Pairwise comparisons were performed by unpaired Student $t$-tests with Welch's correction for unequal variances and by one-way analysis of variance (ANOVA) followed by Tukey test for post-hoc pairwise comparisons. Significance level was set at 95\% ( $\mathrm{p}<0.05)$ in all analysis.

\section{Results}

3.1. Both CMC2.24 and Curcumin reduce the inflammatory infiltrate and preserve collagen content in the gingival tissues

Administration of both CMC2.24 and Curcumin caused a significant reduction of the inflammatory cell infiltrate, including both polymorphonuclear cells and mononuclear cells (Figs. $1 \mathrm{a}-\mathrm{i}$ and $2 \mathrm{a}-\mathrm{d}$ ). However, the decrease in the number of mononuclear cells in the curcumin-treated animals was not statistically significant. In contrast, administration of CMC2.24 potently reduced the inflammatory infiltrate by reducing both polymorphonuclear and mononuclear cells. Stereometric analysis indicated that administration of CMC2.24, but not Curcumin, significantly increased the proportion of collagen fibers in the gingival tissues in comparison with that of the positive control animals. This observation, may be related to an increased proliferation and/or activity of extracellular matrix synthesis by fibroblasts in the region or, alternatively, by reduced degradation of pre-existing collagen, which may be associated with attenuation of the inflammatory response, including greater potency of CMC2.24, compared to curcumin, as an MMP (including collagenases) inhibitor (see Discussion). 

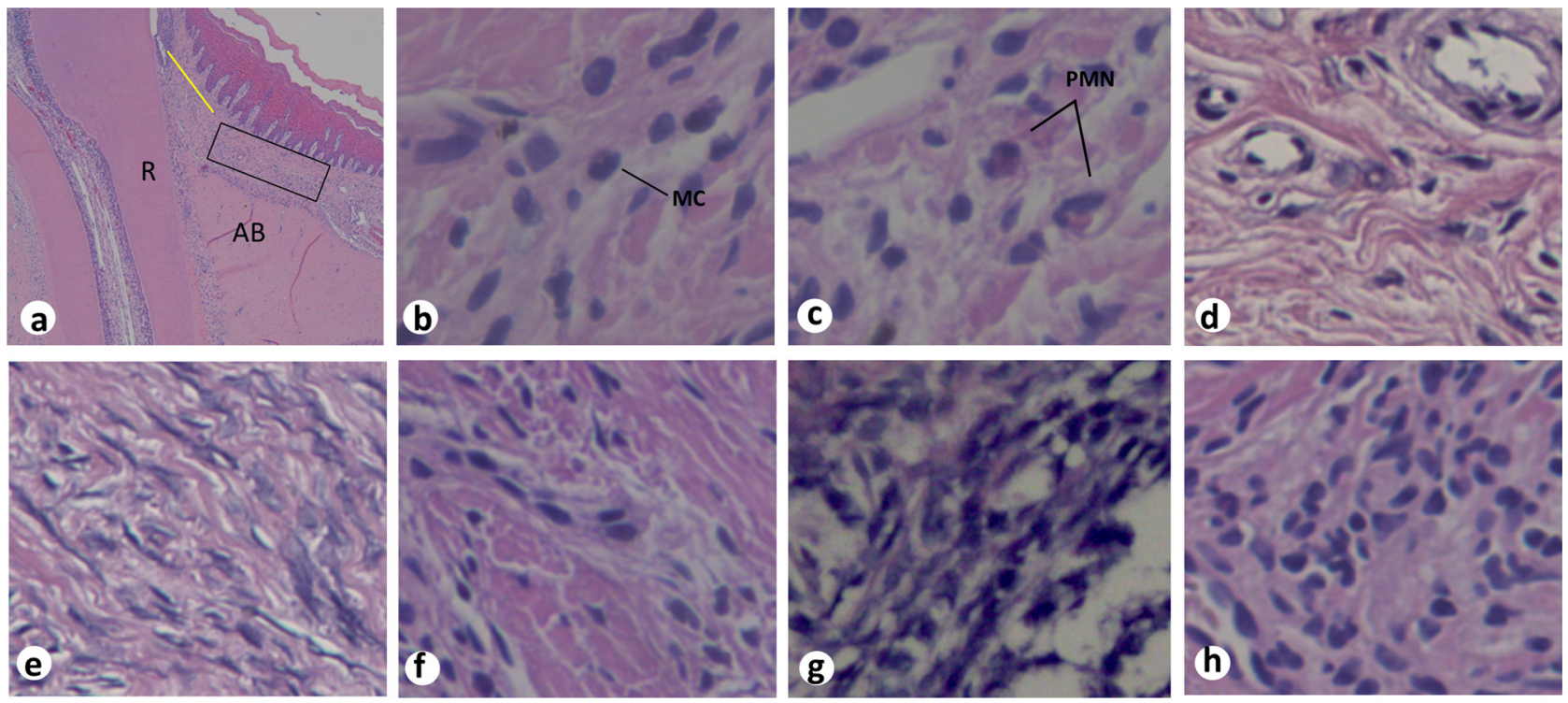

Fig. 1. Histological characteristics of H/E-stained gingival tissues subjected to LPS-induced periodontal disease according to the experimental groups. (a) Palatal region where the injections of LPS were performed, corresponding to the rectangular box. (d) The contralateral side (control) was injected with the same volume of vehicle (PBS) in the same region (d, at higher magnification). (e) Intense inflammatory in gingival tissues produced by LPS injections. (f) CMC2.24 treated animals in comparison to (g) CMC2.24-vehicle control, (h) Curcumin treated animals. Images were obtained at $200 \times$ magnification. High power magnification $(600 \times)$ images detail the morphological characteristics of mononuclear (b) and PMNs cell (c) as they were considered for differential counting. The area of interest is located at $400 \mu \mathrm{m}$ of the tooth (distance represented by the yellow line in image a), corresponding to the site of injections and the bulk of inflammatory infiltrate. AB, alveolar bone. R, root. PMN, polymorphonuclear neutrophil. MC, mononuclear cell. (For interpretation of the references to colour in this figure legend, the reader is referred to the web version of this article.)

\subsection{Oral administration of CMC2.24, but not Curcumin, significantly reduces alveolar bone loss and osteoclastogenesis associated with LPS- induced periodontal disease}

Microcomputer tomograph analysis indicates that only CMC2.24 attenuated inflammatory bone resorption (Fig. 3). Carboxymethylcellulose vehicle had no effect, and specimens from the corn oil-treated animals were not scanned because our research group has a previous study showing that this vehicle does not affect the extent of inflammatory bone resorption in this model (Guimaraes et al., 2012). The main purpose of this study was to compare the effects of CMC2.24 and natural curcumin on alveolar bone resorption associated with the LPS-induced model of periodontal disease in an experiment performed concomitantly, using experimental rats of the same strain. Of primary importance, the number of TRAP-positive multinucleated cells, identified by immunohistochemistry, was significantly decreased only in the CMC2.24-treated group, which supports the interpretation that the bone-sparing effects of this compound observed in the $\mu \mathrm{CT}$ analysis are due, at least in part, to the inhibition of osteoclastogenesis in vivo. Curcumin had no effect on the number of osteoclasts present in the region of interest (Fig. 4A-C).

\subsection{Curcumin, but not CMC2.24, reduces apoptosis}

Curcumin has a pro-apoptotic effect, which is relevant for its anticancer properties (Pfeffer \& Singh, 2018). However, increased apoptosis in inflammation may generate a loop of sustained activation of the immune response, as dying cells release damage-associated molecular patterns (DAMPs). Inflammation induced by LPS injections increased apoptosis in the periodontal microenvironment, as indicated by significant increases of TUNEL-positive and active caspase-3-stained cells in the gingival tissues. This increase in apoptosis was slight and nonstatistically significant in the alveolar bone crest (osteocytes). Administration of curcumin, but not of CMC2.24, potently and significantly reduced the number of apoptotic cells in the gingival tissues and also of osteocytes in the alveolar bone crest, both in the presence and absence of LPS-induced inflammation. The number of active caspase 3-stained cells was slightly reduced by both CMC2.24 and curcumin, but this change did not reach statistical significance, suggesting that curcumin attenuates late-stage apoptosis. (Fig. 5a-d).

\section{Discussion}

Oral administration of both CMC2.24 and curcumin significantly inhibited the local inflammatory response in the LPS-induced periodontal disease model. But only CMC2.24 reduced inflammation-associated bone resorption significantly, which is supported by the significant decrease in the number of osteoclasts in the periodontal tissues of CMC2.24-treated animals. Interestingly, curcumin, but not CMC2.24, reduced late-stage apoptosis in the periodontal microenvironment, suggesting differential biological effects for these compounds in the doses used in this study.

Curcumin has documented potent anti-inflammatory effects in several disorders (Bundy, Walker, Middleton, \& Booth, 2004; Deodhar, Sethi, \& Srimal, 1980; Garcea et al., 2004; Holt, Katz, \& Kirshoff, 2005; Hanai et al., 2006; Heng, Song, Harker, \& Heng, 2000; Sharma et al., 2004; Sharma et al., 2001), including periodontal diseases (Correa et al., 2017; Guimaraes et al., 2011; Guimaraes et al., 2012; Zhou et al., 2013). However, its effects on inflammatory bone resorption are still controversial, since the variability in the activity of this compound is due, mostly, to its poor pharmacological properties, and poor absorption in the gastrointestinal tract (Sharma, Gescher, \& Steward, 2005). Other possibilities could include weaker direct effects on osteoclast activity and MMPs.

In fact, chemically modified curcumin (CMC2.24) is more soluble than curcumin in water-based vehicles and this compound has three zinc-binding moieties that are able to inhibit matrix metalloproteinases, which are essential in the degradation of connective tissue, including bone tissue. The increased bioavailability of CMC2.24 may be at least partly responsible for the attenuation of bone resorption; however the decrease on the osteoclast numbers observed in CMC2.24-treated animals suggest an effect on osteoclast differentiation. 
(a)

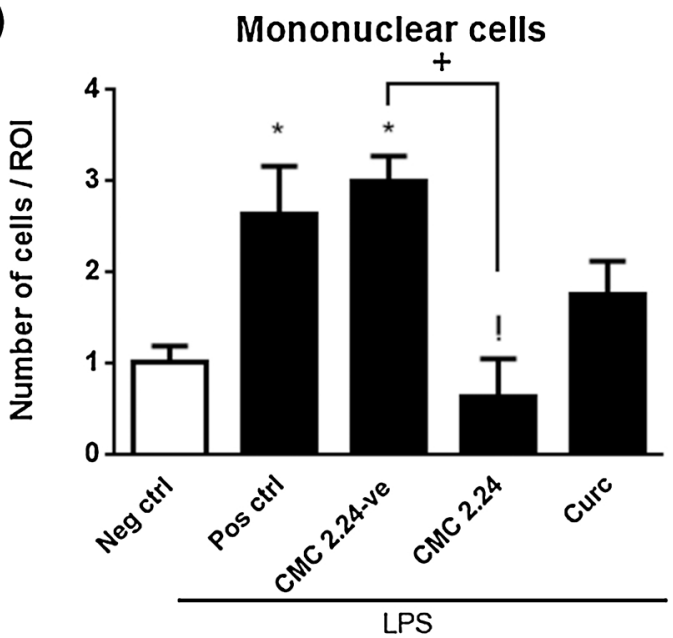

(c)

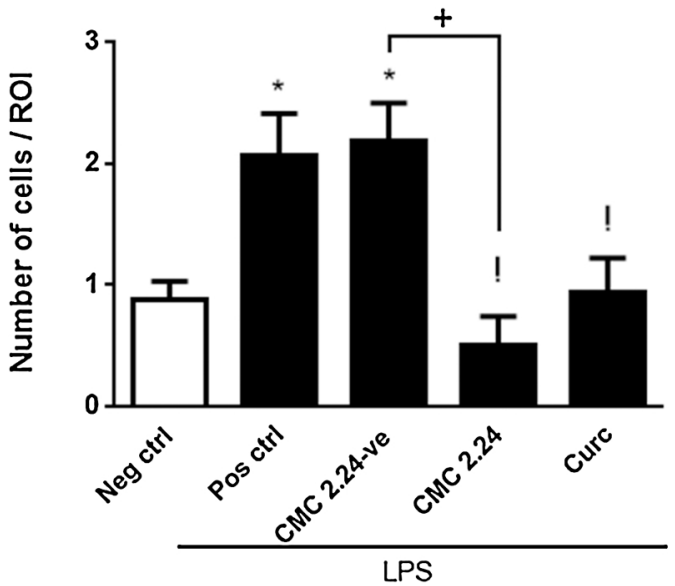

(b)

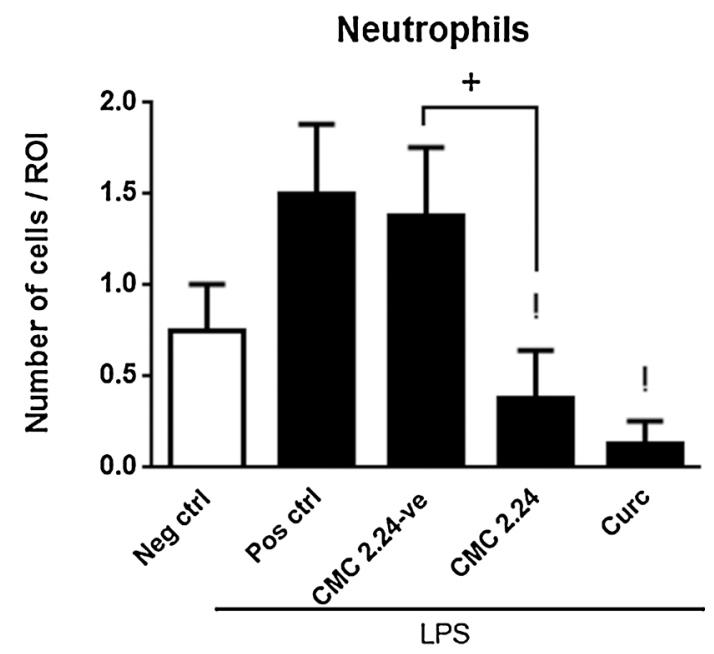

(d)

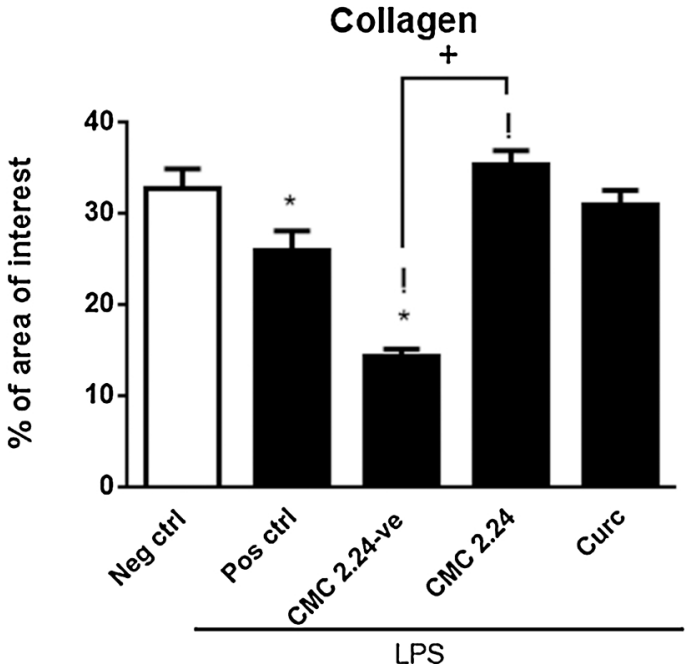

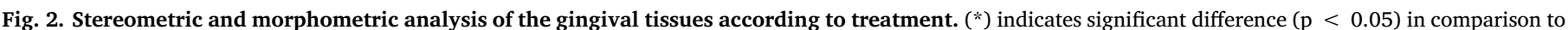

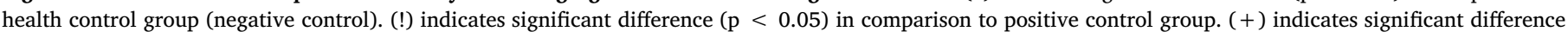
( $p<0.05$ ) between the groups. Bars indicate means and vertical lines standard error of mean.

The reduction in the number of end-stage (TUNEL-positive) apoptotic cells in the gingival tissue and alveolar bone (osteocytes) only in animals treated with curcumin shows that the compounds seem to act through different mechanisms, These differences may be a reflection of significant structural differences between curcumin and CMC2.24 (e.g., diketonic curcumin vs. tri-ketonic CMC2.24). Apoptotic cells release damage-associated moleculas patterns (DAMPs) that may further ctivate innate immune receptors and stimulate efferocytosis by phagocytosing immune cells. Moreover, apoptosis of inflammatory cells is an important mechanism in the resolution of the inflammation, and its modulation by chemotherapeutic agents is a strategy in promoting the resolution of chronic inflammatory processes. Several studies have evaluated the therapeutic potential of curcumin in modulating apoptosis of neutrophils (Jancinova et al., 2011; Jancinova et al., 2009; Prasad, Raghavendra, Lokesh, \& Naidu, 2004). Interestingly, the proapoptotic effect of curcumin on various neoplastic cells in vitro is widely documented in support to its anti-cancer effects and involves various mechanisms, including microRNA (Lu et al., 2017), inhibition of NF-kB (Schwertheim et al., 2017), regulation of pro- (p21, Bad, Bax, Bid, Bim p53, caspase-3, caspase-9) and anti-apoptotic (Bcl-2, Bcl-w) genes (Montazeri et al., 2016; Zhou et al., 2017), increase of $\mathrm{Ca}^{2+}$ influx (Seo, Kim, Dhanasekaran, Tsang, \& Song, 2016), inhibition of Akt/ FOXO1 signaling (Liou, Chen, \& Yang, 2017). In contrast, in vivo, oral administration of curcumin reduced neuronal cell apoptosis in a preclinical model of kindling (Saha, Chakrabarti, Kumari, Bhatia, \& Banerjee, 2016) and also in a model of reperfusion injury by inhibiting NF-kB, caspase-3 and caspase-9 (Li, Suwanwela, \& Patumraj, 2017). Curcumin administered by intraperitoneal injections suppressed oxidative-stress-associated apoptosis of photoreceptor cells in the eye of rats (Emoto et al., 2013). These discrepancies may be associated with variations in the type of study (in vitro vs in vivo), experimental model, dose of curcumin, administration routes, vehicle used and preparation of curcumin; but may also be associated with a time-dependent response to curcumin. In cardiomyoblasts, concomitant treatment of curcumin with exposure to a stress-inducer potentiated the ROS-associated apoptosis, whereas pre-treatment of curcumin attenuated the apoptotic effect of the stress inducer (Jain \& Rani, 2017). Curcumin pretreatment also inhibited particulate matter-induced apoptosis of endothelial cells (Shi, Deng, \& Zhang, 2017) and diabetes-associated apoptosis of splenocytes (Rashid, Chowdhury, Ghosh, \& Sil, 2017) by inhibiting production of ROS and caspase 3 activity. Interestingly, CMC2.24 did not have a consistent effect on apoptosis indicated by TUNEL assay or expression of active caspase 3.

It is important to consider the limitations of our analysis, as we cannot discriminate if the apoptotic cells in the gingival tissues are resident, stromal cells or inflammatory cells. It is tempting to speculate 


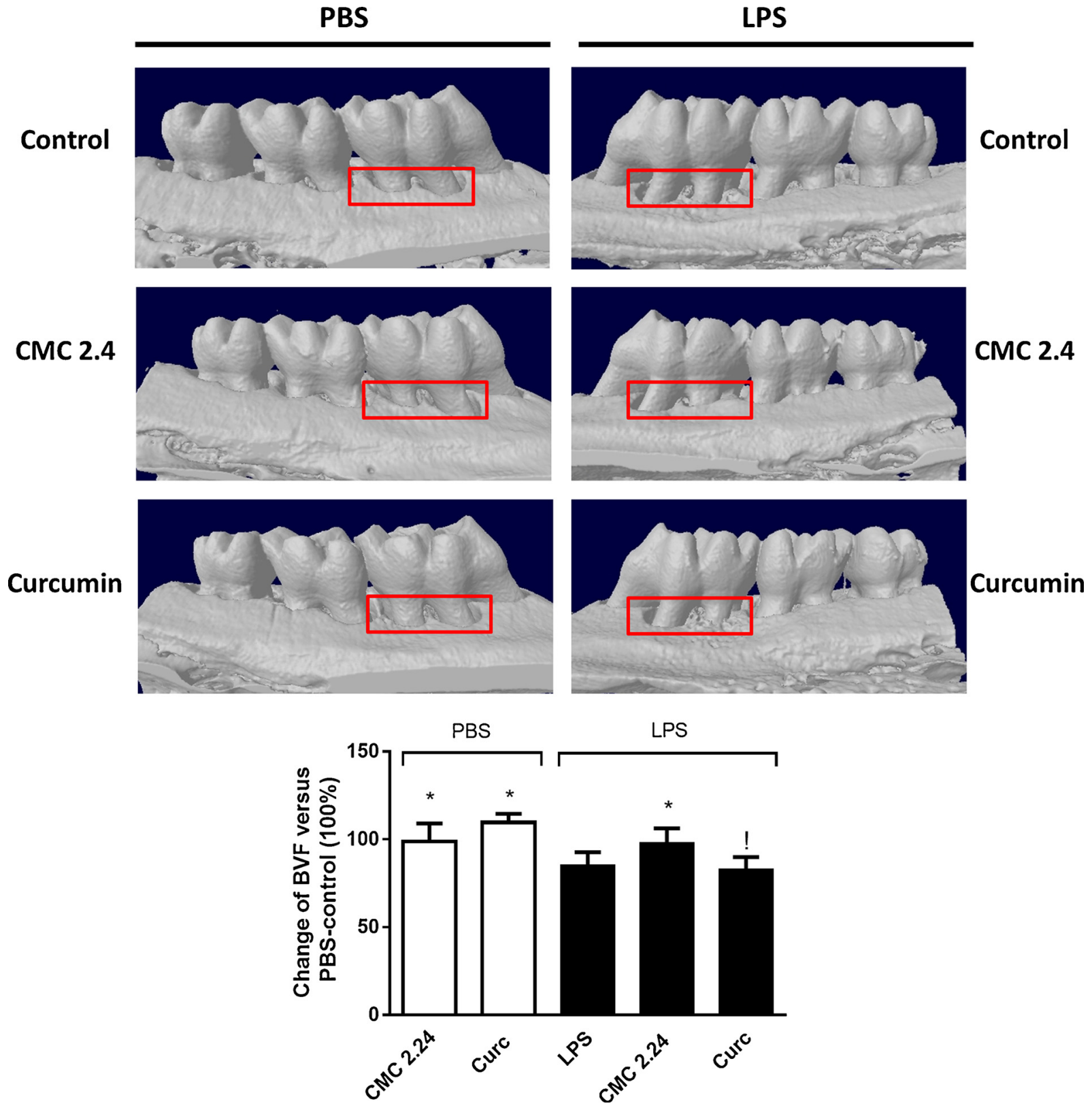

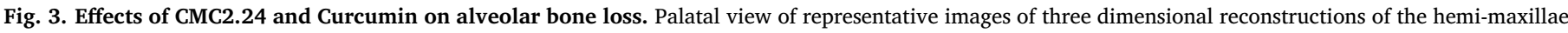

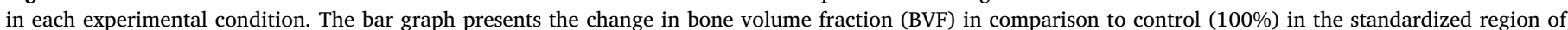

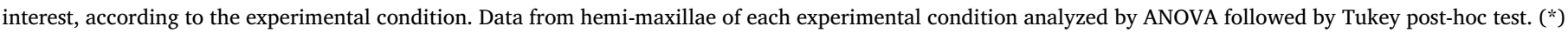

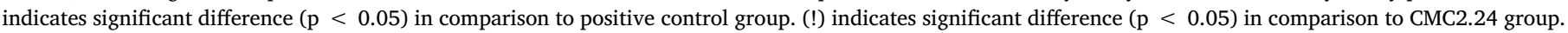

that increased apoptosis of inflammatory cells may represent a resolving process, with reduced levels of pro-inflammatory mediators. On the other hand, tissue damage associated with progression of periodontal disease can also be accelerated and healing impaired by increased apoptosis of stromal cells, such as fibroblasts and endothelial cells. The increase in collagen content suggests that the former possibility is more likely.

Interestingly, curcumin and CMC2.24 present similar anti-inflammatory effects, which may be derived from their MMP-inhibiting properties. However, these compounds may have distinct bone-sparing properties, as the decrease in the apoptosis of osteocytes observed only with administration of curcumin may also be bone-protective since apoptotic osteocytes are associated with increased bone resorption and secretion of RANKL (Cabahug-Zuckerman et al., 2016). In hormonalrelated osteoporosis or corticosteroid-associated bone resorption, apoptosis of osteocytes is particularly critical. Corticosteroids induce apoptosis of mature osteocytes impairing the secretion of soluble mediators required for physiological bone remodeling and triggering the production of osteoclast-inducing and activating RANKL by apoptotic osteocytes (Cabahug-Zuckerman et al., 2016; Jilka \& O'Brien, 2016; Komori, 2016). Intriguingly, the reduction in apoptotic osteocytes did not attenuate inflammatory bone resorption or osteoclastogenesis in our study. However, we cannot rule out the possibility that higher doses of curcumin may effectively decrease bone resorption in this model.

A previous study by our research group (Guimaraes et al., 2012) had already demonstrated that oral administration of curcumin $(100 \mathrm{mg} /$ $\mathrm{Kg}$ /day) did not reduce bone resorption in the ligature-induced periodontal disease model. However, similarly to the findings in this study, curcumin significantly reduced the inflammatory infiltrate and potently inhibited gene expression of inflammatory mediators IL-6, TNF- $\alpha$ and prostaglandin E2synthase (murine homologous of COX-2). Using the 
(A)

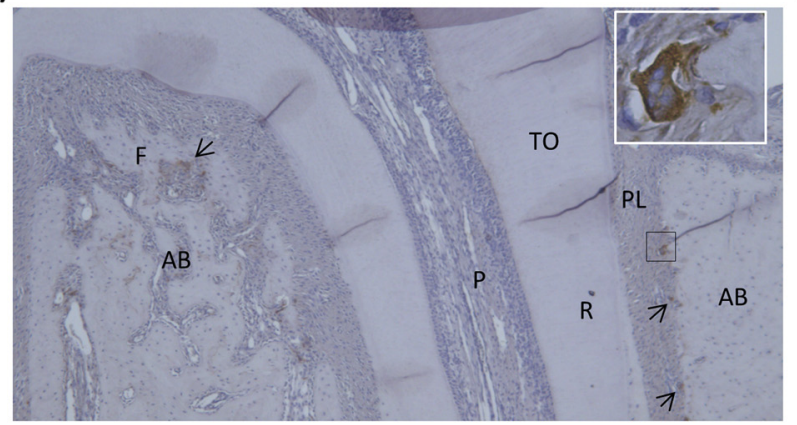

(C)

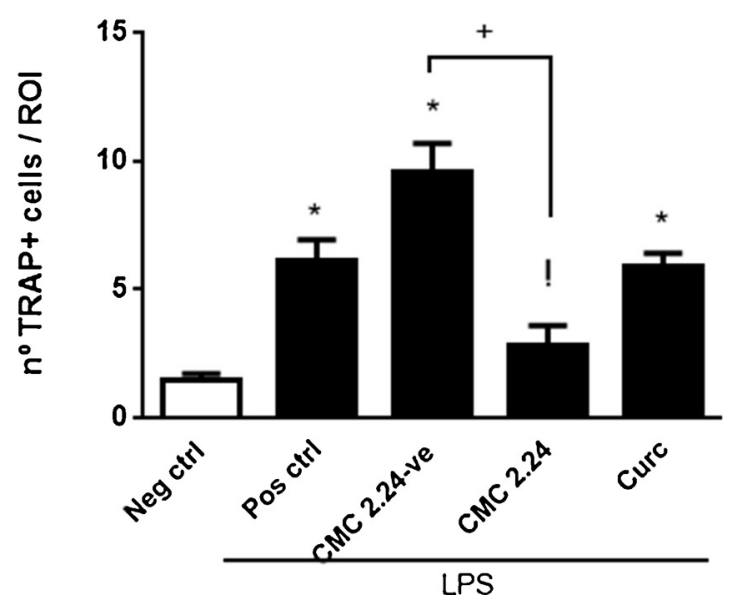

(B)
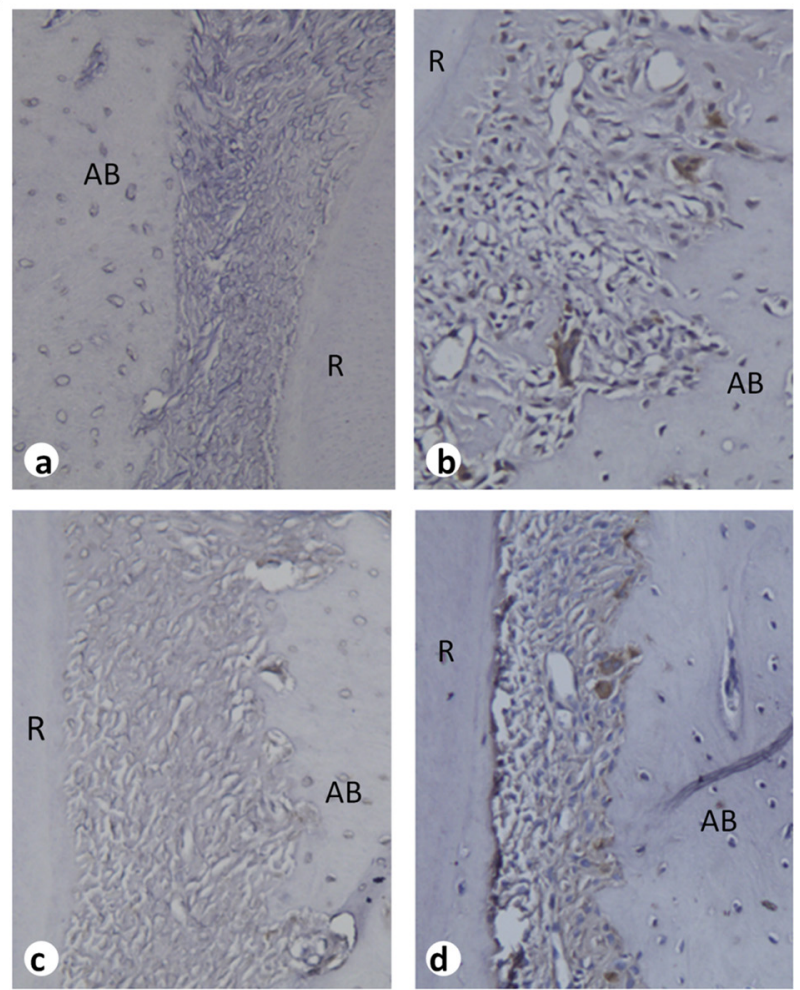

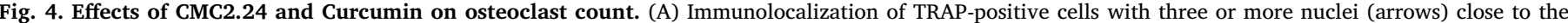

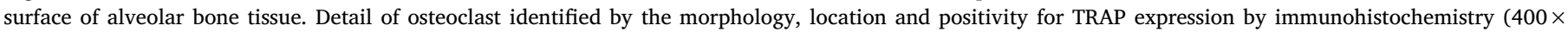

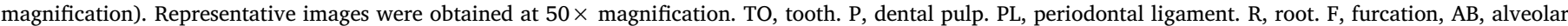

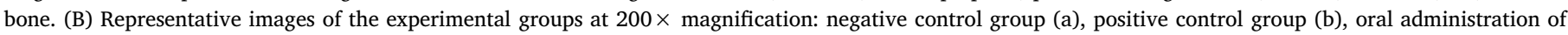

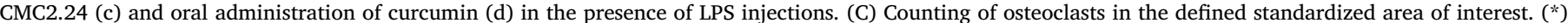

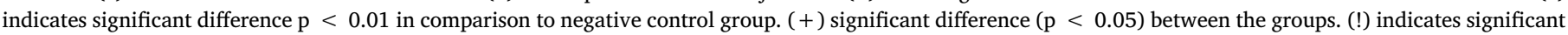

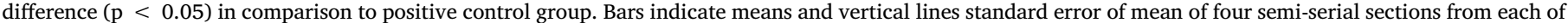
five different animals in each experimental group.

same ligature-induced periodontal disease model and same dose of orally administered curcumin $(100 \mathrm{mg} / \mathrm{Kg} /$ day $)$ for a longer trial period (30 days), Zhou et al., 2013 detected a significant reduction in IL-6 and TNF- $\alpha$ levels, and in contrast to our findings, there was a reduction of inflammatory bone resorption. The differences between this report (Zhou et al., 2013) and our previous study can be attributed to variations in the experimental protocol, including the evaluation period (15 vs 30 days), form of assessment ( $\mu$ CT vs morphometric evaluation), the vehicle used for compound dilution (corn oil vs. ethanol 9\%), and the lineage of experimental animals used (Holtzman vs Wistar rats).

In the present study, the lack of significant effect of curcumin on the inflammatory bone resorption was confirmed in LPS-induced periodontal disease model, and for the first time compared in the same experiment to CMC2.24, a synthetic curcumin analogue, which significantly inhibited both bone resorption and osteoclastogenesis. Indeed, much of the variability in the literature regarding the use of curcumin may be influenced by the fact that 'natural' curcumin is not chemically pure, but rather a mixture of several related chemical species (the three most prevalent are: curcumin, demethoxicurcumin and bis-demethoxicurcumin). Variation in the proportion of these chemical species in distinct commercial preparations of 'curcumin' may account for varying biological effects; as opposed to a chemically defined synthetic compound such as CMC2.24, which may yield a more consistent biological response.

In summary, oral administration of CMC2.24, but not curcumin, significantly reduced inflammatory bone resorption and the number of osteoclasts in the proximity of the alveolar bone. On the other hand, curcumin, but not CMC2.24, significantly inhibited apoptosis in the gingival tissue and also of osteocytes, both in the presence or absence of inflammation.

\section{Conflict of interests}

All authors declare that they have no conflict of interest.

\section{Funding}

Financial support provided by FAPESP $<$ gn $1>2010 / 19660$ $2</$ gn $1>$.

\section{Ethical approval}

All the experimental protocols were approved by the Ethical Committee for Animal Use (CEUA) of the School of Dentistry at Araraquara -UNESP (12/2011) and in accordance with the guidelines from the Brazilian College for Animal Experimentation (COBEA).

\section{Acknowledgments}

Financial support provided by FAPESP 2010/19660-2. The authors 
(a)

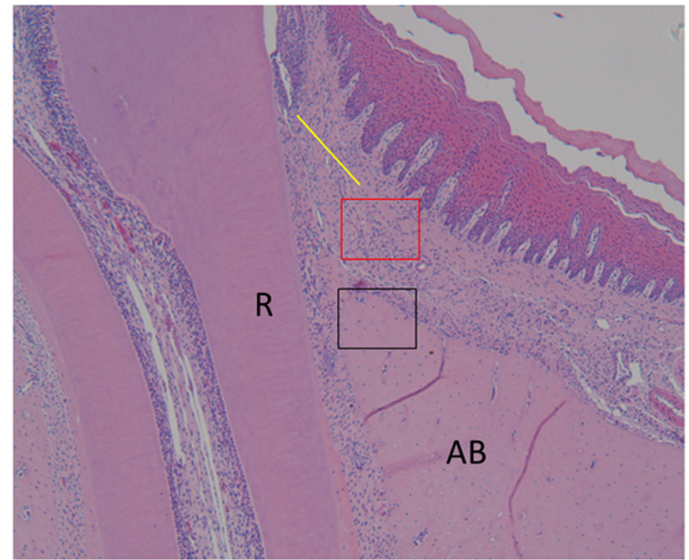

(c)

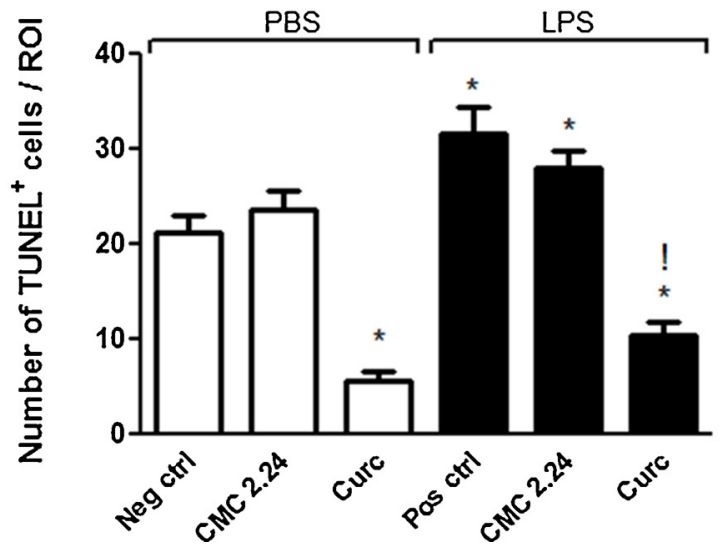

(b)

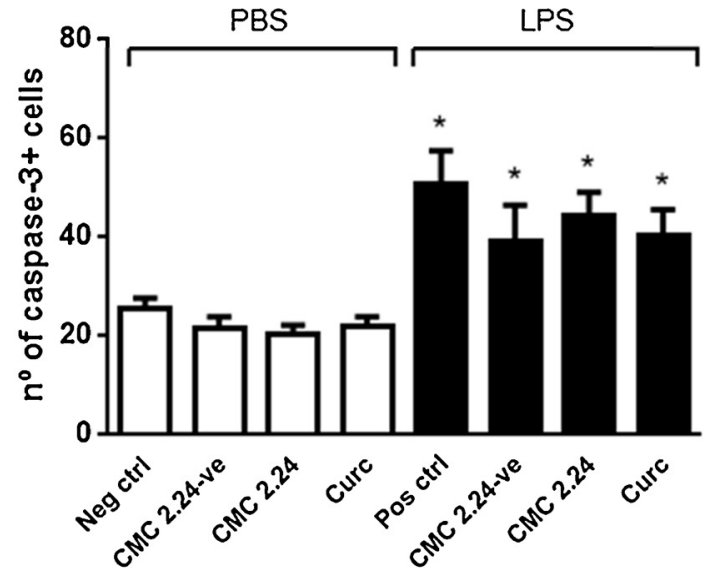

(d)

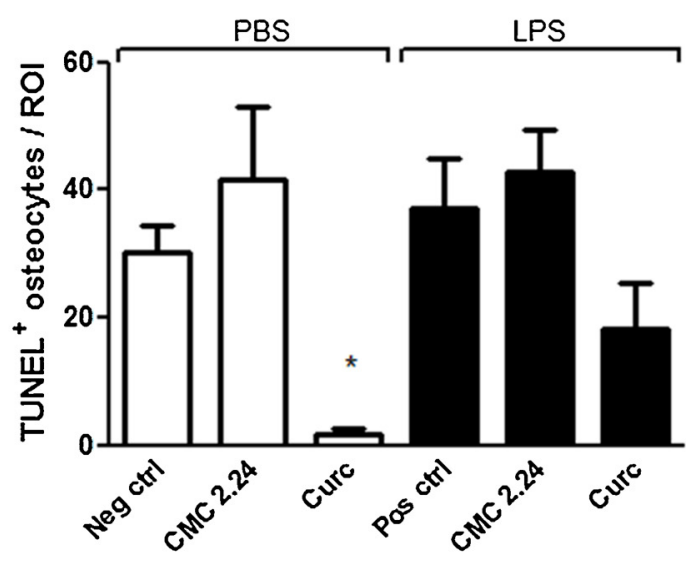

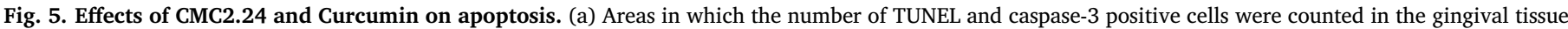

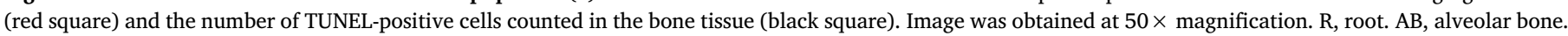

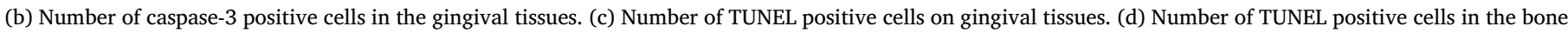

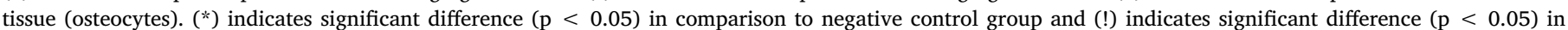

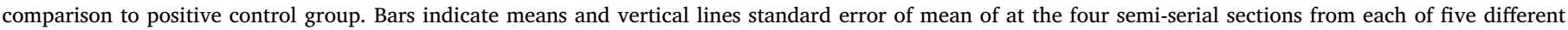

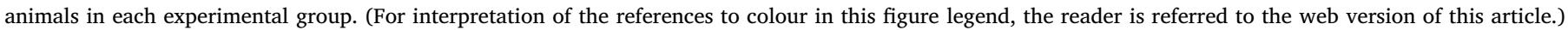

are very grateful to Mrs. Ana Claudia Gregolin Costa Miranda (senior laboratory assistant, Department of Diagnosis and Surgery, School of Dentistry at Araraquara-UNESP) for her support in the histological processing and staining and to Ms. Luana Carla Pires (laboratory technician, Department of Diagnosis and Surgery, School of Dentistry at Araraquara-UNESP), operator of the $\mu \mathrm{CT}$ instrument.

\section{References}

Anand, P., Kunnumakkara, A. B., Newman, R. A., \& Aggarwal, B. B. (2007). Bioavailability of curcumin: Problems and promises. Molecular Pharmaceutics, 4(6), 807-818. http://dx.doi.org/10.1021/mp700113r.

Bundy, R., Walker, A. F., Middleton, R. W., \& Booth, J. (2004). Turmeric extract may improve irritable bowel syndrome symptomology in otherwise healthy adults: A pilot study. Journal of Alternative and Complementary Medicine, 10(6), 1015-1018. http:// dx.doi.org/10.1089/acm.2004.10.1015.

Cabahug-Zuckerman, P., Frikha-Benayed, D., Majeska, R. J., Tuthill, A., Yakar, S., Judex, S., \& Schaffler, M. B. (2016). Osteocyte apoptosis caused by hindlimb unloading is required to trigger osteocyte RANKL production and subsequent resorption of cortical and trabecular bone in mice femurs. Journal of Bone and Mineral Research, 31(7), 1356-1365. http://dx.doi.org/10.1002/jbmr.2807.

Correa, M. G., Pires, P. R., Ribeiro, F. V., Pimentel, S. Z., Casarin, R. C., Cirano, F. R., ... Casati, M. Z. (2017). Systemic treatment with resveratrol and/or curcumin reduces the progression of experimental periodontitis in rats. Journal of Periodontal Research, 52(2), 201-209. http://dx.doi.org/10.1111/jre.12382.

Dentino, A., Lee, S., Mailhot, J., \& Hefti, A. F. (2013). Principles of periodontology. Periodontology 2000, 61(1), 16-53. http://dx.doi.org/10.1111/j.1600-0757.2011. 00397.x.

Deodhar, S. D., Sethi, R., \& Srimal, R. C. (1980). Preliminary study on antirheumatic activity of curcumin (diferuloyl methane). Indian Journal of Medical Research, 71, 632-634.

Di Martino, R. M., Luppi, B., Bisi, A., Gobbi, S., Rampa, A., Abruzzo, A., \& Belluti, F. (2017). Recent progress on curcumin-based therapeutics: A patent review (2012-2016). Part I: Curcumin. Expert Opinion on Therapeutic Patents, 27(5), 579-590. http://dx.doi.org/10.1080/13543776.2017.1276566.

Elburki, M. S., Rossa, C., Guimaraes, M. R., Goodenough, M., Lee, H. M., Curylofo, F. A., \& Golub, L. M. (2014). A novel chemically modified curcumin reduces severity of experimental periodontal disease in rats: Initial observations. Mediators of Inflammation, 959471. http://dx.doi.org/10.1155/2014/959471.

Elburki, M. S., Moore, D. D., Terezakis, N. G., Zhang, Y., Lee, H. M., Johnson, F., \& Golub, L. M. (2017). A novel chemically modified curcumin reduces inflammation-mediated connective tissue breakdown in a rat model of diabetes: Periodontal and systemic effects. Journal of Periodontal Research, 52(2), 186-200. http://dx.doi.org/10.1111/ jre.12381.

Elburki, M. S., Rossa, C., Jr., Guimaraes-Stabili, M. R., Lee, H. M., Curylofo-Zotti, F. A., Johnson, F., \& Golub, L. M. (2017). A chemically modified curcumin (CMC 2.24) inhibits nuclear factor kappaB activation and inflammatory bone loss in murine models of LPS-induced experimental periodontitis and diabetes-associated natural periodontitis. Inflammation, 40(4), 1436-1449. http://dx.doi.org/10.1007/s10753017-0587-4.

Emoto, Y., Yoshizawa, K., Uehara, N., Kinoshita, Y., Yuri, T., Shikata, N., \& Tsubura, A. (2013). Curcumin suppresses N-methyl-N-nitrosourea-induced photoreceptor apoptosis in Sprague-Dawley rats. In Vivo, 27(5), 583-590.

Garcea, G., Jones, D. J., Singh, R., Dennison, A. R., Farmer, P. B., Sharma, R. A., ... Berry, D. P. (2004). Detection of curcumin and its metabolites in hepatic tissue and portal blood of patients following oral administration. British Journal of Cancer, 90(5), 1011-1015. http://dx.doi.org/10.1038/sj.bjc.6601623.

Golub, L. M., Suomalainen, K., \& Sorsa, T. (1992). Host modulation with tetracyclines and their chemically modified analogues. Current Opinion in Dentistry, 2, 80-90.

Graves, D. T., Kang, J., Andriankaja, O., Wada, K., \& Rossa, C., Jr. (2012). Animal models to study host-bacteria interactions involved in periodontitis. Frontiers of Oral Biology, 
15, 117-132. http://dx.doi.org/10.1159/000329675.

Guimaraes, M. R., Coimbra, L. S., de Aquino, S. G., Spolidorio, L. C., Kirkwood, K. L., \& Rossa, C., Jr. (2011). Potent anti-inflammatory effects of systemically administered curcumin modulate periodontal disease in vivo. Journal of Periodontal Research, 46(2), 269-279. http://dx.doi.org/10.1111/j.1600-0765.2010.01342.x.

Guimaraes, M. R., de Aquino, S. G., Coimbra, L. S., Spolidorio, L. C., Kirkwood, K. L., \& Rossa, C., Jr. (2012). Curcumin modulates the immune response associated with LPSinduced periodontal disease in rats. Innate Immunity, 18(1), 155-163. http://dx.doi. org $/ 10.1177 / 1753425910392935$.

Hajishengallis, G., Lamont, R. J., \& Graves, D. T. (2015). The enduring importance of animal models in understanding periodontal disease. Virulence, 6(3), 229-235. http://dx.doi.org/10.4161/21505594.2014.990806.

Hanai, H., Iida, T., Takeuchi, K., Watanabe, F., Maruyama, Y., Andoh, A., \& Koide, Y. (2006). Curcumin maintenance therapy for ulcerative colitis: Randomized, multicenter, double-blind, placebo-controlled trial. Clinical Gastroenterology and Hepatology, 4(12), 1502-1506. http://dx.doi.org/10.1016/j.cgh.2006.08.008.

Heng, M. C., Song, M. K., Harker, J., \& Heng, M. K. (2000). Drug-induced suppression of phosphorylase kinase activity correlates with resolution of psoriasis as assessed by clinical, histological and immunohistochemical parameters. British Journal of Dermatology, 143(5), 937-949.

Holt, P. R., Katz, S., \& Kirshoff, R. (2005). Curcumin therapy in inflammatory bowel disease: A pilot study. Digestive Diseases and Sciences, 50(11), 2191-2193. http://dx. doi.org/10.1007/s10620-005-3032-8.

Jain, A., \& Rani, V. (2017). Mode of treatment governs curcumin response on doxorubicin-induced toxicity in cardiomyoblasts. Molecular and Cellular Biochemistry, 442, 81-96. http://dx.doi.org/10.1007/s11010-017-3195-.

Jancinova, V., Perecko, T., Nosal, R., Kostalova, D., Bauerova, K., \& Drabikova, K. (2009). Decreased activity of neutrophils in the presence of diferuloylmethane (curcumin) involves protein kinase C inhibition. European Journal of Pharmacology, 612(1-3), 161-166. http://dx.doi.org/10.1016/j.ejphar.2009.03.080.

Jancinova, V., Perecko, T., Nosal, R., Mihalova, D., Bauerova, K., \& Drabikova, K. (2011). Pharmacological regulation of neutrophil activity and apoptosis: Contribution to new strategy for modulation of inflammatory processes. Interdisciplinary Toxicology, 4(1), 11-14. http://dx.doi.org/10.2478/v10102-011-0003-0.

Jilka, R. L., \& O'Brien, C. A. (2016). The role of osteocytes in age-related bone loss. Current Osteoporosis Reports, 14(1), 16-25. http://dx.doi.org/10.1007/s11914-016-0297-0.

Komori, T. (2016). Glucocorticoid signaling and bone biology. Hormone and Metabolic Research, 48(11), 755-763. http://dx.doi.org/10.1055/s-0042-110571.

Kumar, A., Ahuja, A., Ali, J., \& Baboota, S. (2010). Conundrum and therapeutic potential of curcumin in drug delivery. Critical Reviews in Therapeutic Drug Carrier Systems, 27(4), 279-312.

Lao, C. D., Ruffin, M. T.t., Normolle, D., Heath, D. D., Murray, S. I., Bailey, J. M., .. Brenner, D. E. (2006). Dose escalation of a curcuminoid formulation. BMC Complementary and Alternative Medicine, 6, 10. http://dx.doi.org/10.1186/14726882-6-10

Li, W., Suwanwela, N. C., \& Patumraj, S. (2017). Curcumin prevents reperfusion injury following ischemic stroke in rats via inhibition of NFkappaB, ICAM-1, MMP-9 and caspase-3 expression. Molecular Medicine Reports, 16(4), 4710-4720. http://dx.doi. org/10.3892/mmr.2017.7205.

Liou, A. T., Chen, M. F., \& Yang, C. W. (2017). Curcumin induces p53-Null hepatoma cell line Hep3B apoptosis through the AKT-PTEN-FOXO4 pathway. Evidence-Based Complementary and Alternative Medicine, (2017), 4063865. http://dx.doi.org/10. $1155 / 2017 / 4063865$

Lu, Y., Wang, J., Liu, L., Yu, L., Zhao, N., Zhou, X., \& Lu, X. (2017). Curcumin increases the sensitivity of Paclitaxel-resistant NSCLC cells to Paclitaxel through microRNA30c-mediated MTA1 reduction. Tumour Biology, 39(4), http://dx.doi.org/10.1177/ 10104283176983531010428317698353.

Montazeri, M., Sadeghizadeh, M., Pilehvar-Soltanahmadi, Y., Zarghami, F., Khodi, S. Mohaghegh, M., ... Zarghami, N. (2016). Dendrosomal curcumin nanoformulation modulate apoptosis-related genes and protein expression in hepatocarcinoma cell lines. International Journal of Pharmaceutics, 509(1-2), 244-254. http://dx.doi.org/ 10.1016/j.ijpharm.2016.05.039.

Pfeffer, C. M., \& Singh, A. T. K. (2018). Apoptosis: A target for anticancer therapy. International Journal of Molecular Sciences, 19(2), http://dx.doi.org/10.3390/ ijms19020448.

Prasad, N. S., Raghavendra, R., Lokesh, B. R., \& Naidu, K. A. (2004). Spice phenolics inhibit human PMNL 5-lipoxygenase. Prostaglandins Leukot Essent Fatty Acids, 70(6), 521-528. http://dx.doi.org/10.1016/j.plefa.2003.11.006.

Rashid, K., Chowdhury, S., Ghosh, S., \& Sil, P. C. (2017). Curcumin attenuates oxidative stress induced NFkappaB mediated inflammation and endoplasmic reticulum dependent apoptosis of splenocytes in diabetes. Biochemical Pharmacology, 143, 140-155. http://dx.doi.org/10.1016/j.bcp.2017.07.009.

Saha, L., Chakrabarti, A., Kumari, S., Bhatia, A., \& Banerjee, D. (2016). Antiapoptotic and neuroprotective role of Curcumin in Pentylenetetrazole (PTZ) induced kindling model in rat. Indian Journal of Experimental Biology, 54(2), 133-141.

Schwertheim, S., Wein, F., Lennartz, K., Worm, K., Schmid, K. W., \& Sheu-Grabellus, S. Y. (2017). Curcumin induces G2/M arrest, apoptosis, NF-kappaB inhibition, and expression of differentiation genes in thyroid carcinoma cells. Journal of Cancer Research and Clinical Oncology, 143(7), 1143-1154. http://dx.doi.org/10.1007/ s00432-017-2380-z.

Seo, J. A., Kim, B., Dhanasekaran, D. N., Tsang, B. K., \& Song, Y. S. (2016). Curcumin induces apoptosis by inhibiting sarco/endoplasmic reticulum Ca2 + ATPase activity in ovarian cancer cells. Cancer Letters, 371(1), 30-37. http://dx.doi.org/10.1016/j. canlet.2015.11.021.

Sharma, R. A., McLelland, H. R., Hill, K. A., Ireson, C. R., Euden, S. A., Manson, M. M., .. Steward, W. P. (2001). Pharmacodynamic and pharmacokinetic study of oral Curcuma extract in patients with colorectal cancer. Clinical Cancer Research, 7(7), 1894-1900.

Sharma, R. A., Euden, S. A., Platton, S. L., Cooke, D. N., Shafayat, A., Hewitt, H. R., \& Steward, W. P. (2004). Phase I clinical trial of oral curcumin: Biomarkers of systemic activity and compliance. Clinical Cancer Research, 10(20), 6847-6854. http://dx.doi. org/10.1158/1078-0432. CCR-04-0744.

Sharma, R. A., Gescher, A. J., \& Steward, W. P. (2005). Curcumin: The story so far. European Journal of Cancer, 41(13), 1955-1968. http://dx.doi.org/10.1016/j.ejca. 2005.05.009.

Shehzad, A., Park, J. W., Lee, J., \& Lee, Y. S. (2013). Curcumin induces radiosensitivity of in vitro and in vivo cancer models by modulating pre-mRNA processing factor 4 (Prp4). Chemico-Biological Interactions, 206(2), 394-402. http://dx.doi.org/10.1016/ j.cbi.2013.10.007.

Shi, J., Deng, H., \& Zhang, M. (2017). Curcumin pretreatment protects against PM2.5induced oxidized lowdensity lipoproteinmediated oxidative stress and inflammation in human microvascular endothelial cells. Molecular Medicine Reports, 16(3), 2588-2594. http://dx.doi.org/10.3892/mmr.2017.6935.

Shoba, G., Joy, D., Joseph, T., Majeed, M., Rajendran, R., \& Srinivas, P. S. (1998). Influence of piperine on the pharmacokinetics of curcumin in animals and human volunteers. Planta Medica, 64(4), 353-356. http://dx.doi.org/10.1055/s-2006957450.

Vernillo, A. T., Ramamurthy, N. S., Golub, L. M., \& Rifkin, B. R. (1994). The nonantimicrobial properties of tetracycline for the treatment of periodontal disease. Current Opinion in Periodontology, 111-118.

Zhang, Y., Golub, L. M., Johnson, F., \& Wishnia, A. (2012). pKa, zinc- and serum albuminbinding of curcumin and two novel biologically-active chemically-modified curcumins. Current Medicinal Chemistry, 19(25), 4367-4375.

Zhou, T., Chen, D., Li, Q., Sun, X., Song, Y., \& Wang, C. (2013). Curcumin inhibits inflammatory response and bone loss during experimental periodontitis in rats. Acto Odontologica Scandinavica, 71(2), 349-356. http://dx.doi.org/10.3109/00016357. 2012.682092.

Zhou, Q. M., Sun, Y., Lu, Y. Y., Zhang, H., Chen, Q. L., \& Su, S. B. (2017). Curcumin reduces mitomycin $\mathrm{C}$ resistance in breast cancer stem cells by regulating Bcl-2 familymediated apoptosis. Cancer Cell International, 17, 84. http://dx.doi.org/10.1186/ s12935-017-0453-3. 